\title{
Clock-controlled mir-142-3p can target its activator, Bmal1
}

\author{
Xiaochao Tan ${ }^{1}$, Peng Zhang ${ }^{2}$, Lan Zhou ${ }^{2}$, Bin Yin ${ }^{1}$, Hui Pan ${ }^{3^{*}}$ and Xiaozhong Peng ${ }^{1 *}$
}

\begin{abstract}
Background: microRNAs (miRNAs) are shown to be involved in the regulation of circadian clock. However, it remains largely unknown whether miRNAs can regulate the core clock genes (Clock and Bmal1).

Results: In this study, we found that mir-142-3p directly targeted the 3'UTR of human BMAL1 and mouse Bmal1. The over-expression (in 293ET and NIH3T3 cells) and knockdown (in U87MG cells) of mir-142-3p reduced and up-regulated the Bmal1/BMAL1 mRNA and protein levels, respectively. Moreover, the expression level of mir-142-3p oscillated in serum-shocked NIH3T3 cells and the results of ChIP and luciferase reporter assays suggested that the expression of mir-142-3p was directly controlled by CLOCK/BMAL1 heterodimers in NIH3T3 cells.

Conclusions: Our study demonstrates that mir-142-3p can directly target the 3'UTR of Bmal1. In addition, the expression of mir-142-3p is controlled by CLOCK/BMAL1 heterodimers, suggesting a potential negative feedback loop consisting of the miRNAs and the core clock genes. These findings open new perspective for studying the molecular mechanism of circadian clock.
\end{abstract}

Keywords: mir-142-3p, Bmal1, Circadian clock

\section{Background}

Daily patterns of physiological and behavioral processes can be observed in almost all organisms, ranging from cyan bacterium to humans. The circadian clock plays important roles in local and systemic physiology and in pathology [1-3]. The molecular machinery of the circadian clock is thought to be composed of self-sustaining transcriptional feedback loops. The core proteins of the molecular pathway regulating the circadian clock are CLOCK and BMAL1 $[4,5]$, which form heterodimers that drive the transcription of circadian output genes (CCGs) [6,7]. The disruption of the expression of these two core molecules leads to remarkable changes in the circadian clock. The mutation of the Clock gene (Clock $\Delta 19 / 19)$ or the deletion of the Bmall gene (Bmal1-/-) abolishes circadian oscillations [4,5]. However, Clock knock-out mice exhibit a normal physiological rhythm because of

\footnotetext{
*Correspondence: ph3610@gmail.com; pengxiaozhong@pumc.edu.cn ${ }^{3}$ Department of Endocrinology, Peking Union Medical Hospital, Chinese Academy of Medical Sciences and Peking Union Medical College, Beijing 100730, China

'State Key Laboratory of Medical Molecular Biology, Institute of Basic Medical Sciences, Chinese Academy of Medical Sciences and Peking Union Medical College, Beijing 100005, China

Full list of author information is available at the end of the article
}

compensation by NPAS2 [8,9]. CCGs, such as Per and Cry, can weaken the transcriptional activity of CLOCK/ BMAL1 heterodimers, forming a negative feedback loop called the core loop [10]. Rev-Erb $\alpha$ and ROR $\alpha$ bind to an ROR element in the promoter of Bmal1, inhibiting or activating its transcription, respectively, which is called the stabilizing loop [11].

MicroRNAs (miRNAs) are small non-coding RNA molecules that regulate the expression of target genes at the post-transcriptional level. The roles of miRNAs in the circadian clock have been discussed in several reports. Xu et al. showed that the levels of specific miRNAs (e.g., mir-96, mir-124a, mir-103, mir-182 and mir106b) oscillate in the mouse retina in a circadian pattern [12]. Clock-controlled mir-219 acts in the fine tuning of the length of the circadian period, and light-induced mir-132 was shown to be a negative regulator of the light-dependent resetting of the clock [13]. The mir-192/ 194 cluster was identified as a potent inhibitor of the Per gene family, the enforced expression of which leads to an altered circadian rhythm [14]. Kadener et al. found that a miRNA, the developmental regulator bantam, plays a role in the core circadian pacemaker by targeting clock in Drosophila [15]. In addition, mir-206 was shown 
to be a regulator of the circadian clock in skeletal muscle [16]. Recently, a report showed that mir-494 and mir142-3p, two circulating miRNAs, can target the Bmal1 3' UTR in mice [17]. Thus, mounting evidence suggests that miRNAs act as very important regulators of the circadian clock [18].

We are interested in the miRNAs which can directly target the core clock regulators, Clock and Bmal1, and prediction, followed by experimental validation, was performed to identify potential Clock/Bmal1-targeting miRNAs. The level of CLOCK protein remains nearly unchanged during the circadian cycle [19], and it can be functionally compensated by NPAS2. Therefore, we focused on the potential Bmal1-targeting miRNAs. Here in this study, we found that mir-142-3p directly targeted Bmal1 and its expression was regulated by CLOCK/ BMAL1 heterodimers.

\section{Results}

\section{Mir-142-3p is a Bmal1-targeting miRNA}

By computational prediction using three algorithmic methods (TargetScan, PicTar and MicroCosm), we found two miRNAs that putatively target Bmall (Figure 1A). These two miRNAs (mir-142-3p and mir-448) were subjected to validation by luciferase reporter assays using reporters containing the 3'UTR of mouse Bmal1 or human BMAL1. The mir-142-pcDNA3.1 and mir-448pcDNA3.1 expression plasmids were first transfected into 293ET cells and the expressions of mature miRNAs were verified by quantitative RT-PCR (Additional file 1A). Over-expression of mir-142 but not mir-448 significantly repressed the activity of the Bmal1/BMAL1 3'UTR-luciferase reporter (Figure 1B). We also found that over-expression of mir-142 could significantly reduce the luciferase reporter RNA levels (Additional file $1 \mathrm{~B})$, suggesting that mir-142 was able to accelerate target mRNA degradation. Because pre-mir-142 can produce two mature miRNAs (mir-142-3p and mir-142-5p) (Figure 1C, top), a luciferase reporter assay was performed to ensure that mir-142-3p was the relevant effector. Indeed, the results confirmed that mir-142-3p but not mir-142-5p repressed the Bmal1/BMAL1 3'UTR-luciferase reporter activity (Figure 1C, bottom).

As there are two mir-142-3p binding sites in the 3' UTR of Bmal1, we mutated one or both of them to determine which site was functional, even though the first binding site (position1-7) is poorly conserved among mammals (Figure 1D). In luciferase reporter assays, mutating each of the two mir-142-3p binding sites significantly reduced the repression effect of mir-142 over-expression on luciferase activity, and double mutations (M1 + M2) showed greater effect than single mutation (M1 or M2) (Figure 1E). The luciferase activity of $(\mathrm{M} 1+\mathrm{M} 2)$ was still decreased by about $30 \%$ with mir-
142 over-expression, which may be ascribed to the background of luciferase reporter experiments for that mir142 over-expression also led to an approximately $25 \%$ reduction of luciferase activity of the empty reporter vector (LUC) (Figure 1E). In GFP reporter assays, we got very similar results that each of the binding site was functional for the interaction between mir-142-3p and Bmal13'UTR (Figure 1F).

\section{Mir-142-3p regulates the Bmal1 mRNA and protein levels}

To investigate the regulatory effect of mir-142-3p on the expression of endogenous Bmal1, we detected the expression level of mir-142-3p and Bmal1 protein level in six cell lines (NIH3T3, 293ET, MCF-7, U87MG, T98G and U251) generally used in our laboratory. We found that the level of mir-142-3p was extremely high and the protein level of Bmal1 was low in U87MG cells compared with the other five cell lines. In contrast, NIH3T3, 293ET, MCF-7, T98G and U251 cells with low mir-142$3 p$ levels expressed high levels of Bmal1 protein (Figure 2A). To confirm that mir-142-3p can regulate the expression of Bmal1, mir-142-3p was over-expressed in the NIH3T3 and 293ET cells or knocked down in U87MG cells, and the mRNA and protein levels of human BMAL1 and mouse Bmal1 were determined. The over-expression of mir-142-3p led to reductions in the levels of both Bmal1 (BMAL1) mRNA and protein in NIH3T3 (Figure 2B) and 293ET cells (Figure 2C), and knocking down mir-142-3p with antagomirs increased the expression of BMAL1 mRNA and protein in U87MG cells (Figure 2D). These results showed that mir-142-3p could regulate the expression of Bmal1 by directly targeting its 3'UTR.

\section{Mir-142 expression is under clock control}

We have identified mir-142-3p as a potential regulator of Bmal1; therefore, it is of interest to explore the possibility that if this Bmal1-targeting miRNA is under circadian control. In a synchronized NIH3T3 cell model, we found that the level of mir-142-3p oscillated rhythmically, with the peak levels ( $24 \mathrm{~h}$ and $48 \mathrm{~h}$ ) phase lagging the crests ( $4 \mathrm{~h}$ and $28 \mathrm{~h}$ ) of Bmal1 mRNA (Figure 3), suggesting the expression of mir-142-3p might be under circadian control. Interestingly, by analyzing the 5 ' flank sequence of the mir-142 gene we found a conserved canonical E-box (CACGTG) (Figure 4A). The results of ChIP assays showed that CLOCK was able to bind to the E-box directly; the canonical E-box of Per1 and the upstream sequence of the mir-20a gene were used as the positive and negative controls, respectively (Figure 4B).

For further study, a $1.6 \mathrm{~kb}$ upstream regulatory sequence of mir-142 gene containing the E-box together with pre-mir-142 was then used for a luciferase reporter assay (Figure 5A). Co-expression of Clock and Bmal1 
A

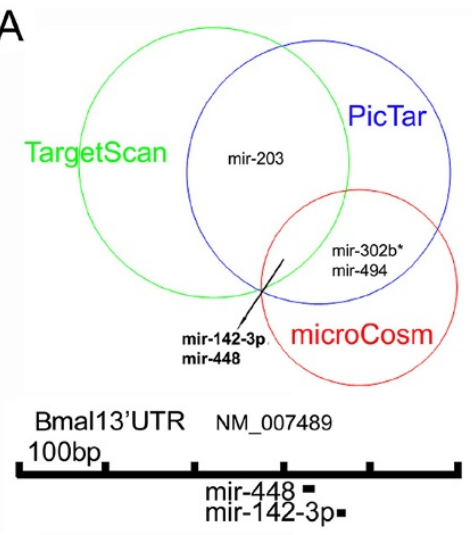

B
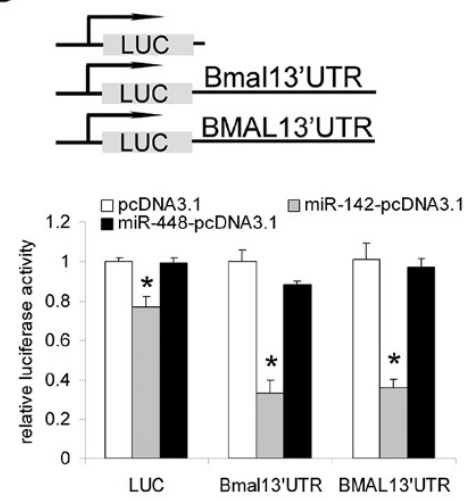

C

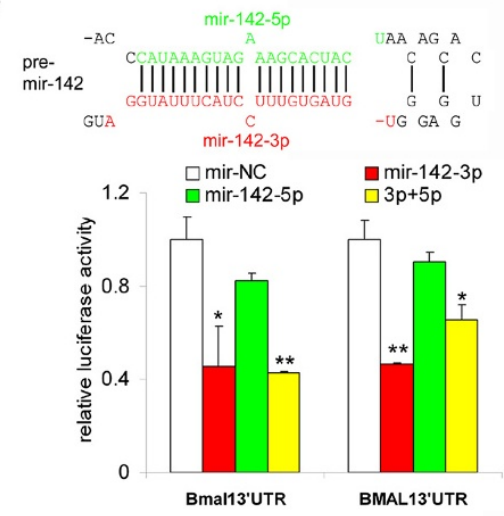

D

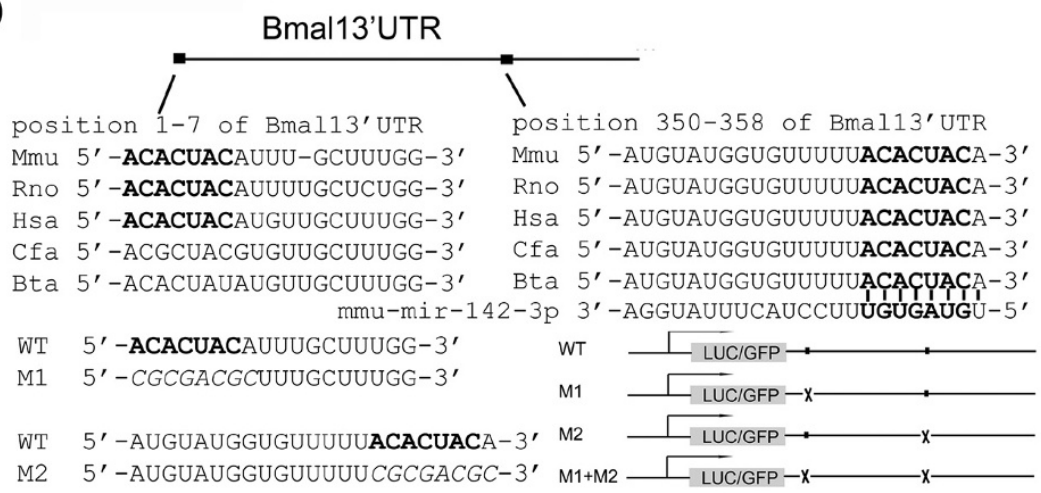

E

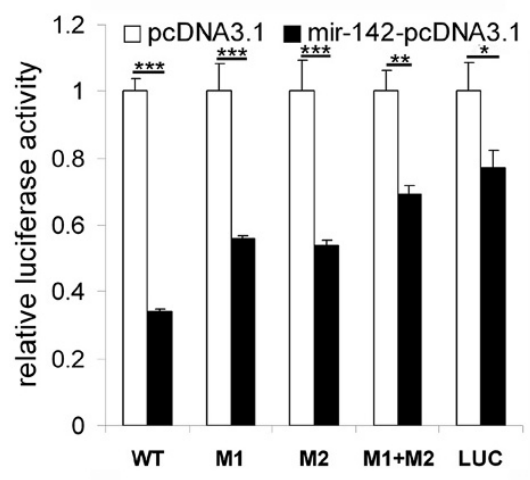

F $1.2 \quad \square$ pcDNA3.1 $\square$ mir-142-pcDNA3.1

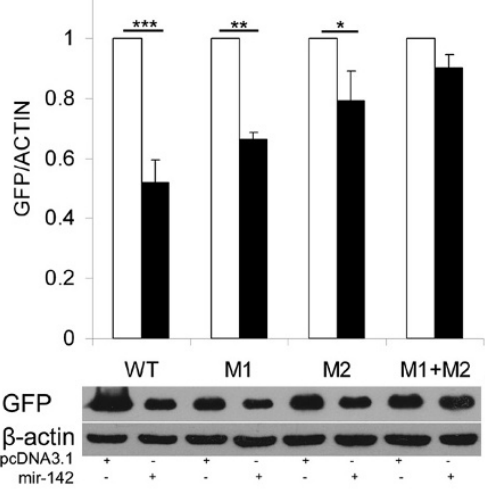

Figure 1 mir-142-3p can target Bmal1 3'UTR. (A) Prediction results using three bioinformatic algorithms (TargetScan, PicTar and MicroCosm). The schematic representation of the binding sites for mir-142-3p and mir-448 in the $3^{\prime \prime}$ UTR of Bmal1 is shown on the bottom. (B) A luciferase reporter assay was employed to screen the miRNAs that could target the mouse Bmal1 and human BMAL1 3' UTR. 293ET cells were co-transfected with luciferase reporter plasmids and mir-142 or mir-448 expression plasmids or control vector, and the normalized firefly luciferase activity was measured (mean $\pm S D, n=3$ ). (C) A luciferase reporter assay was employed to confirm that mir-142-3p but not mir-142-5p can target Bmal1 in 293ET cells. Synthetic mir-142-3p mimics or mir-142-5p mimics or control microRNA mimics (mir-NC) were co-transfected with luciferase reporter plasmids into 293ET cells and the normalized firefly luciferase activity was measured (mean $\pm S D, n=4$ ). The top sketch modified from the miRBase shows that the pre-mir-142 can produce two mature miRNAs, mir-142-3p and -5p. (D) Schematic representation of the mir-142-3p binding sites in the Bmal1 3' UTR and mutation of the mir-142-3p binding sites. In addition to the conserved binding site at position 350-358 of the Bmal1 3' UTR, there is a poorly conserved binding site at position 1-7. (E) 293ET cells were transfected with the empty luciferase reporter vector (LUC) or luciferase reporter plasmids containing the Bmal1 3' UTR with no mutations (WT) or with mutations (M1,M2 and M1 + M2) in the mir-142-3p binding sites and normalized luciferase activity was measured (mean $\pm S D, n=4)$. (F) GFP reporter constructs containing the wild-type Bmal1 3' UTR or mutated sequences were co-transfected into 293ET cells with mir-142-pcDNA3.1 or empty vector (pcDNA3.1), and the expression of GFP was determined by western blotting. The western blotting results were quantified from the pixel values in grayscales $(m e a n \pm S D, n=3)$. Representative western blotting results are shown (bottom). ${ }^{*} P<0.05,{ }^{* *} P<0.01$ and ${ }^{* *} P<0.001$. 


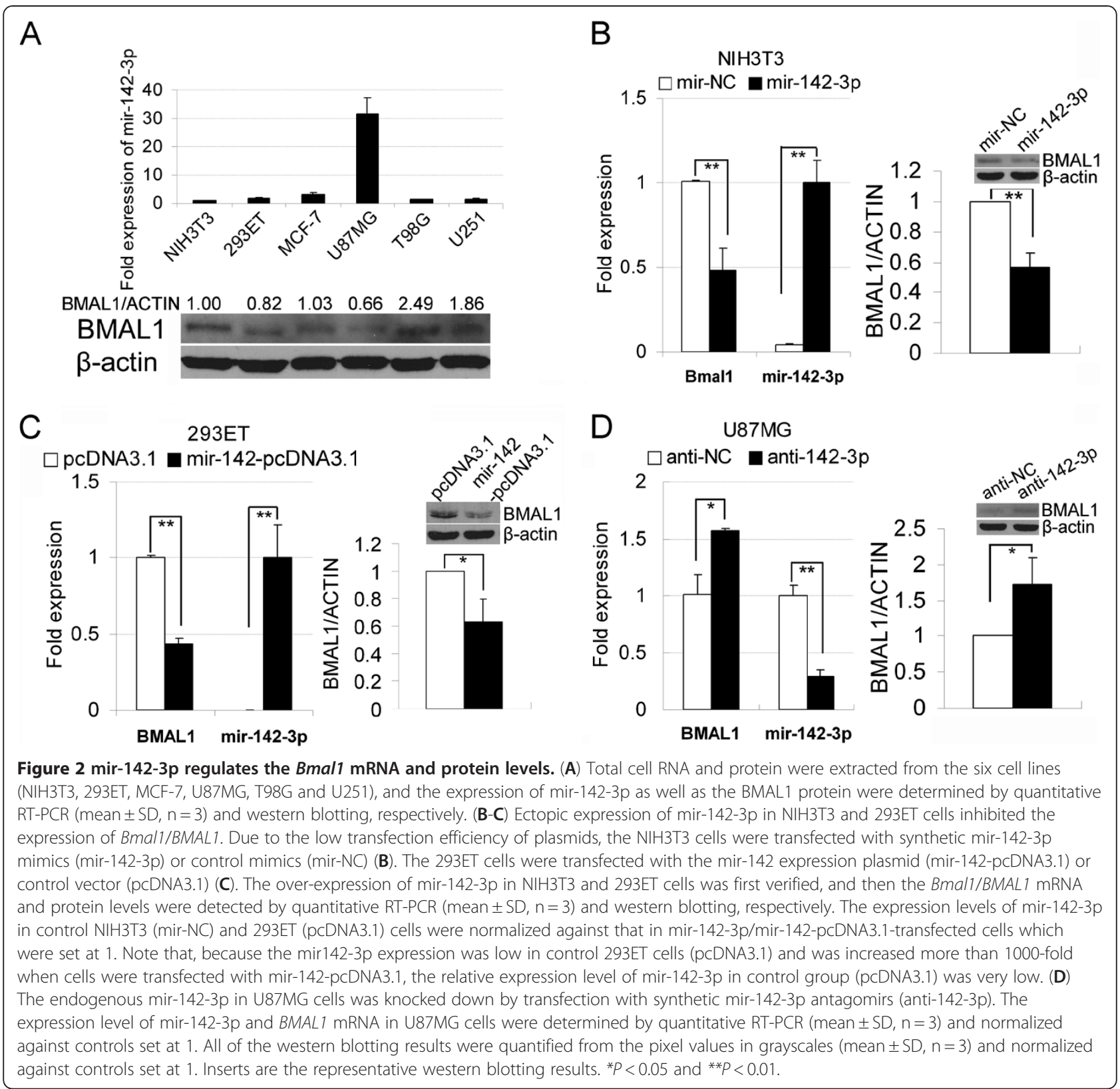

increased the luciferase activity of P142-LUC by approximately 2.5 fold. Mutating the conserved E-box significantly decreased the basal luciferase activity and reduced the induction (1.8 fold) of luciferase activity by coexpressing Clock and Bmal1 (Figure 5B and C). Besides the conserved canonical E-box, there is an unconserved non-canonical E'-box in the upstream regulatory sequence of mir-142 gene which might contribute to the induction of luciferase activity of P142-MT-LUC by coexpression of Clock and Bmal1 (Additional file 2). The results confirmed that the over-expression of Clock and Bmal1 enhanced the transcription of mir-142. Moreover, the simultaneous ectopic expression of Clock and Bmal1 (but not Clock or Bmal1 alone) significantly induced the expression of mir-142-3p in NIH3T3 cells (Figure 5D).

Taken together, CLOCK/BMAL1 heterodimers can bind to the E-box in the upstream regulatory sequence and activate the expression of mir-142; one product of mir-142, mir-142-3p, can act as a negative regulator of Bmal1 by targeting its 3' UTR. A concise model describing this potential negative feedback loop is shown in Figure 5E.

\section{Discussion}

In this study, we showed that the clock-controlled mir142-3p can directly target its circadian activator, Bmal1. 


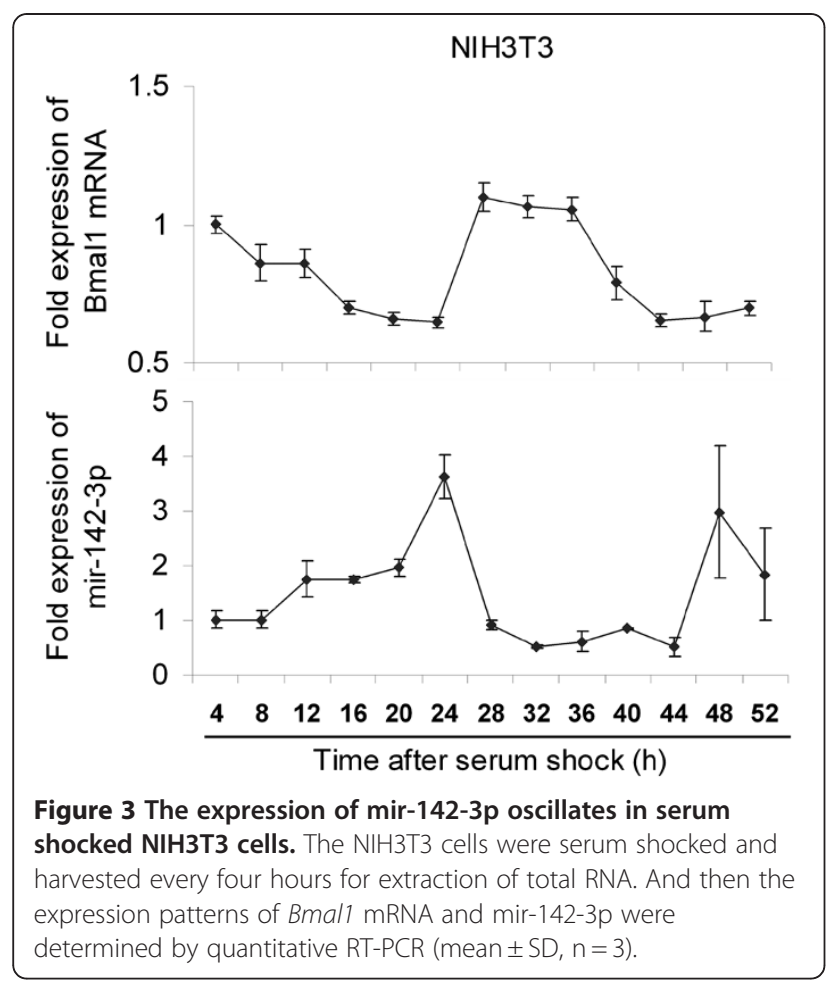

Although mir-142-3p has been reported to be a potential Bmal1-targeting miRNA based on a luciferase reporter assay [17], the present report is the first confirmation that mir-142-3p can directly regulate the expression of Bmal1 both in mouse and human cells. By overexpression of mir-142-3p in NIH3T3 cells, we showed mir-142-3p can regulate the expression of Bmal1. However, a further study on the effects of knocking down mir-142-3p in appropriate murine cell models (with high level of mir-142-3p) will help to confirm the relationship between mir-142-3p and its target at the physiological conditions. Feedback loops formed by clock genes are thought to be the basis of the molecular clock. To our knowledge, this is the first report suggesting a potential negative feedback loop formed by miRNAs and clock genes. Considering the important role of Bmal1 in circadian clock, we speculate that the molecular clock might be also fine tuned by the miRNA-mediated negative feedback.

Besides Bmal1, Clock was also predicted to be targeted by several miRNAs using three bioinformatic algorithms (Additional file 3A). The results of our luciferase reporter assay showed that six candidates (mir-20a, mir106a, mir-106b, mir-148a, mir-182 and mir-301a) could

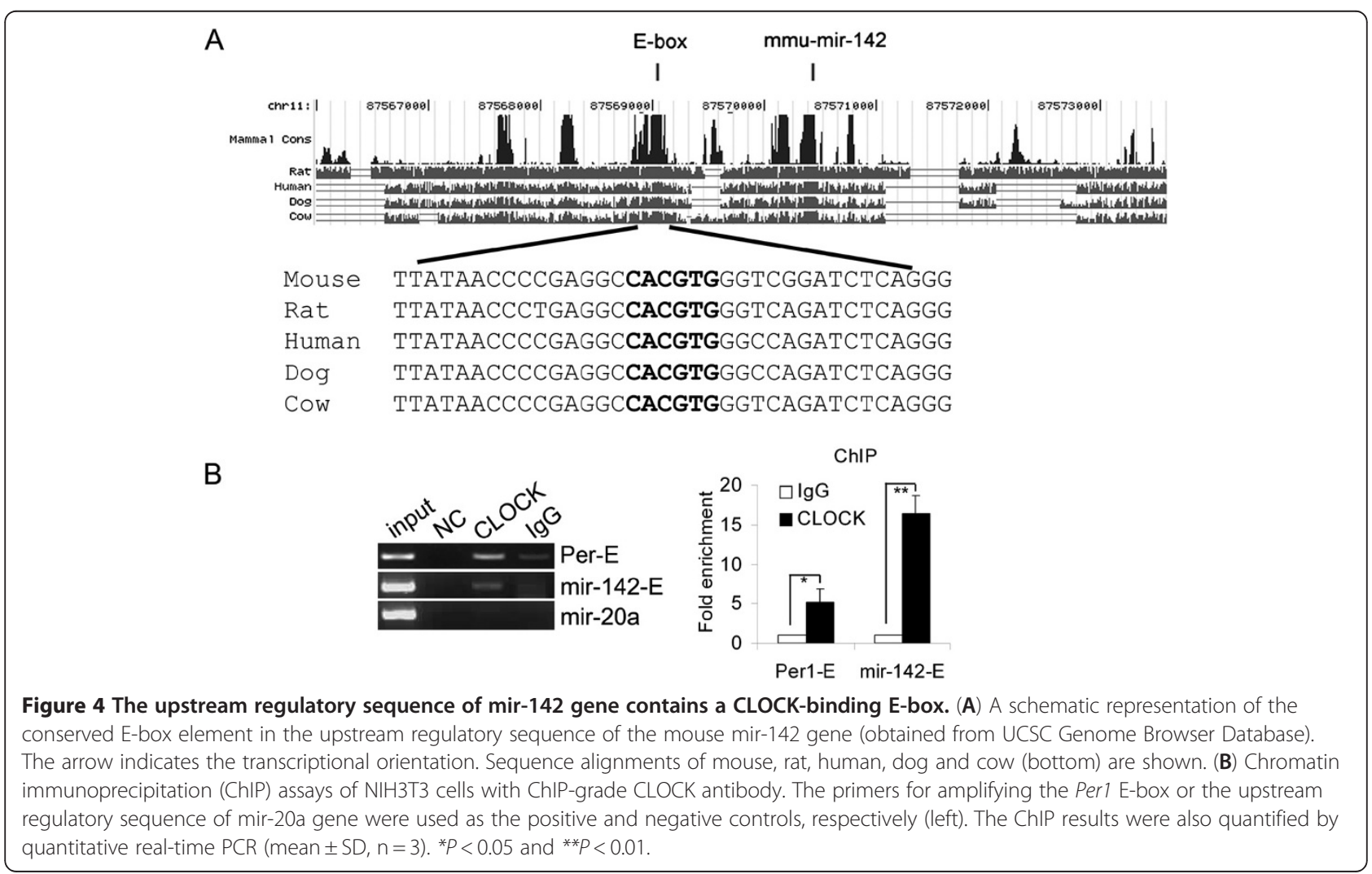




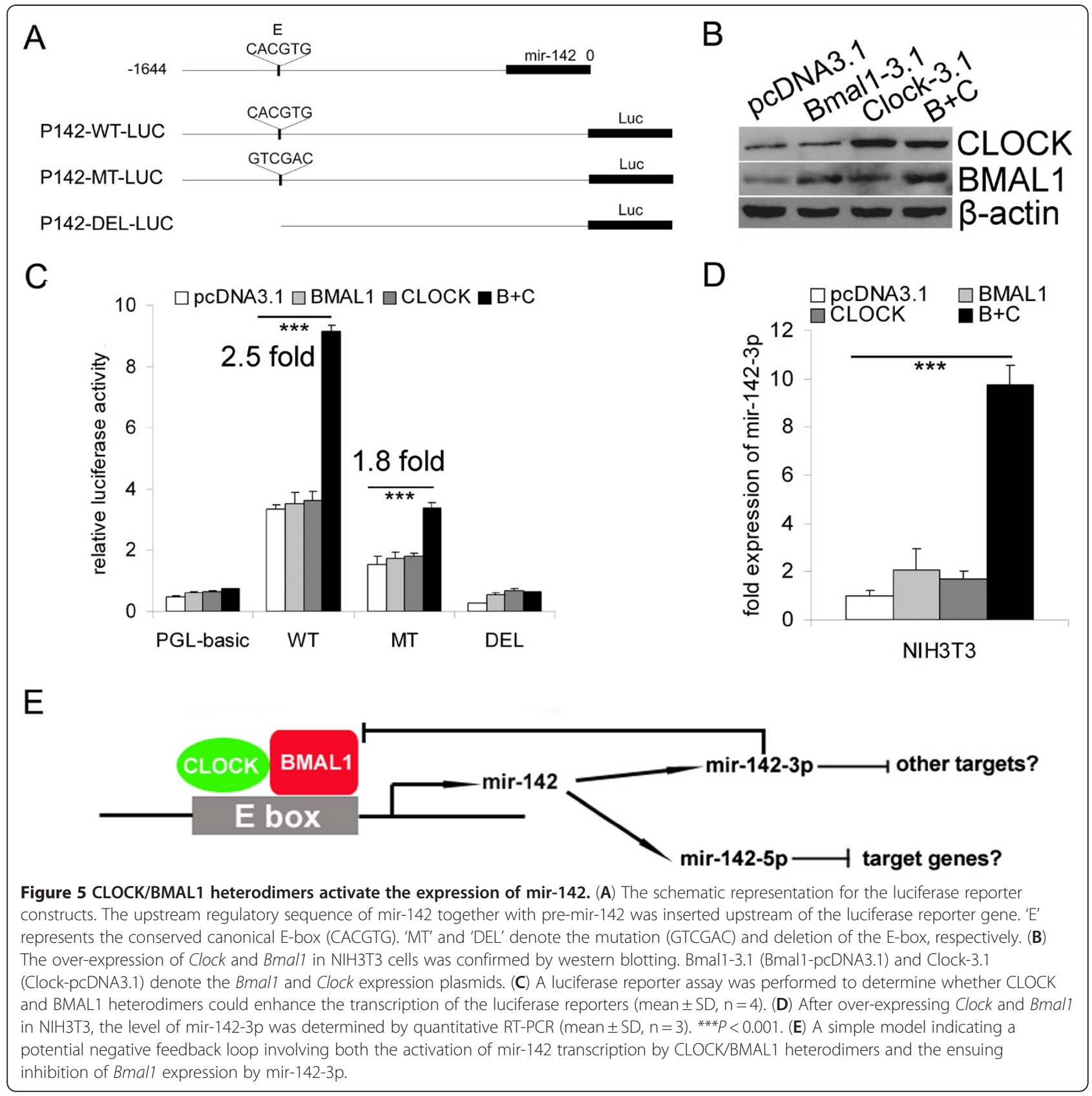

be Clock-targeting miRNAs (Additional file 3B and C). Among them, mir-182 was reported to be a modulator of $C L O C K$ in a recent study [20]. Although we did not perform additional experiments to confirm the interaction between the miRNAs and Clock, the regulation of Clock by miRNAs deserves further study. Indeed, a similar regulatory network consisting of miRNAs and Clock may exist, and a feedback loop involving mir-182/96 and Clock has been hypothesized [12].

In our study, we identified mir-142-3p as a regulator of Bmal1 both in human and mouse cells. Even though we showed that mir-142 is transcriptionally controlled by CLOCK/BMAL1 heterodimers only in a mouse fibroblast cell line (NIH3T3), the E-box in the upstream regulatory sequence of mir-142 gene is perfectly conserved among mammals (Figure 4B). Thus, we hypothesize that this potential negative feedback mechanism could be conserved, at least between mice and human beings.

In our concise model, CLOCK/BMAL1 heterodimers enhance the transcription of mir-142, the product of which, in turn, inhibits the expression of Bmall. In addition to Bmal1, mir-142-3p was reported to target several genes, including $A D C Y$, whose product controls 
cAMP levels [21]. Other genes were also predicted to be targets of mir-142-3p (Additional file 4), and two of them (foxo1 and Nr3c1) are involved in the circadian clock $[22,23]$. The other product of mir-142, mir-142-5p, was also predicted to target numerous genes, the most interesting one of which is Clock, the other master regulator of the molecular clock (Additional file 4). Further studies are needed to confirm these prediction results and to uncover the regulatory role of the mir-142 gene in the circadian clock.

\section{Conclusions}

Our study demonstrates that mir-142-3p can directly target the 3'UTR of Bmal1. In addition, the expression of mir-142-3p is controlled by CLOCK/BMAL1 heterodimers, suggesting a potential negative feedback loop consisting of the miRNAs and the core clock genes. These findings open new perspective for studying the molecular mechanism of circadian clock.

\section{Methods \\ Plasmid DNA constructs}

The mouse Bmal1 and human BMAL1 3'UTRs were cloned from the cDNA of NIH3T3 and 293ET cells, respectively, by PCR. The PCR program was as follows: $95^{\circ} \mathrm{C}$ for $5 \mathrm{~min} ;\left(95^{\circ} \mathrm{C}\right.$ for $30 \mathrm{~s} ; 55^{\circ} \mathrm{C}$ for $30 \mathrm{~s}$ and $72^{\circ} \mathrm{C}$ for $30 \mathrm{~s})$ x30 and $72^{\circ} \mathrm{C}$ for $10 \mathrm{~min}$. The mouse Clock 3' UTR was cloned from cDNA obtained from the NIH3T3 cell line. For the mutagenesis experiment, the mir-142-3p binding site was replaced with a random sequence by bridge PCR. For the miRNA expression plasmids, the genomic sequences of the respective premiRNAs were amplified by PCR from mouse genomic DNA. The PCR program was as follows: $95^{\circ} \mathrm{C}$ for $5 \mathrm{~min}$; $\left(95^{\circ} \mathrm{C}\right.$ for $30 \mathrm{~s} ; 60^{\circ} \mathrm{C}$ for $30 \mathrm{~s}$ and $72^{\circ} \mathrm{C}$ for $\left.30 \mathrm{~s}\right) \times 30$ and $72^{\circ} \mathrm{C}$ for $10 \mathrm{~min}$. The PCR products and respective vectors (pcDNA3.1) were digested directly with restriction enzymes. For cloning the Bmal1/BMAL1 3' UTR, we used the pcDNA3.1-LUC or pcDNA3.1-EGFP vector, and the relevant enzymes were XhoI and XbaI. The pcDNA3.1LUC and pcDNA3.1-EGFP vector are modified from pcDNA3.1 (+) (Invitrogen) as previously described $[24,25]$. For the miRNA expression plasmids, pcDNA3.1 (+) was chosen as the expression vector, and the relevant enzymes were BamHI and XhoI, with the exception of mir-20b (EcoRI and XhoI). All of the primers are listed in (Additional file 5).

\section{Cell culture and transfection}

The 293ET cells were obtained from Dr. Chengyu Jiang (Peking Union Medical College). NIH3T3 was purchased from the American Type Culture Collection (ATCC). All of the cells were maintained in Dulbecco's Modified Eagle Medium supplemented with $10 \%$ fetal bovine serum (FBS), $5 \mathrm{mM}$ L-glutamine, and $100 \mathrm{U} / \mathrm{ml}$ penicillin and $100 \mathrm{mg} / \mathrm{ml}$ streptomycin. Transfections of plasmids, synthetic miRNA mimics and antagomirs were performed with a Lipofectamine 2000 Reagent (Invitrogen, Carlsbad, CA), according to the manufacturers protocol. The sequences of the synthetic mir-142-3p and mir-142-5p mimics are as follows. mir-142-3p: 5'-UG UAGUGUUUCCUACUUUAUGGA-3'; mir-142-5p: 5'-C AUAAAGUAGAAAGCACUACU-3'. The sequence of the synthetic mir-142-3p antagomirs is 5-UCCAUAAAGUA GGAAACACUACA-3'. The synthetic miRNA mimics and antagomirs were purchased from Invitrogen.

\section{Luciferase and GFP reporter assay}

The luciferase assays were performed using the Promega Dual-Luciferase Assay System. For testing the interactions between the Bmal1/Clock 3' UTR and miRNAs, $200 \mathrm{ng}$ of each of the Bmal1/Clock 3' UTR-LUC constructs was co-transfected into 293ET cells with $50 \mathrm{ng}$ of phRL-TK-TK (Renilla luciferase) for normalization and $1 \mu \mathrm{g}$ of miRNA-pcDNA3.1 vector. For the transcriptional activity assay, NIH3T3 cells were transfected with $1.15 \mu \mathrm{g}$ total DNA, including $100 \mathrm{ng}$ of reporter vector (pGL3), $50 \mathrm{ng}$ of phRL-TK, $500 \mathrm{ng}$ of each expression plasmid (Bmal1-pcDNA3.1 or Clock-pcDNA3.1) and pcDNA3.1 to obtain a total amount of 1.15ug. After 48 hours, the cell lysates from all of the treatment groups were collected using Passive Lysis Buffer (Promega). The firefly luciferase activity was analyzed relative to the Renilla luciferase activity in the same sample using a Dual-Luciferase Reporter Assay System (Promega). The luminescence was measured using the GloMax Multi Detection System (Promega). For GFP reporter assay, 293ET cells were co-transfected with $100 \mathrm{ng}$ of GFP reporter construct and $500 \mathrm{ng}$ miRNA expression plasmid or control vector. $48 \mathrm{~h}$ later, cells were harvested for extraction of total RNA or protein. The GFP mRNA and protein level were determined by quantitative RT-PCR and Western blotting, respectively.

\section{Chromatin immunoprecipitation (ChIP) assay}

The ChIP assays were performed as previously described [26]. Briefly, approximately $10^{7}$ NIH3T3 cells were cross-linked by incubation with $1 \%$ formaldehyde for $10 \mathrm{~min}$ at RT, and then the cross-linking reaction was stopped by adding glycine to a final concentration of $0.125 \mathrm{M}$ with continued rocking for $5 \mathrm{~min}$ at room temperature. After washing, the cell pellet was suspended in nuclear lysis buffer, and each cross-linked sample was sonicated on ice and then incubated with anti-CLOCK (Calbiochem, Cat. No. 233170) or IgG. DNA was isolated from the immunoprecipitates and then subjected to PCR using the following primers: Mir-142-E, forward 5'-GTCGCTGGTTTCCTGTCAGT- 
3' and reverse 5'-TGTCCGGAACCTCCACTTAG-3'; Mir-20a-E, forward 5'- TTGTGGTCCTGGCTCTCTC T-3' and reverse 5'- ACTCACCCACTCAGGCAAAC-3'; and mper1-E1, forward 5'- GCTGACTGAGCGG TGTCTGA-3' and reverse 5'- GAGCGCCCTCCA TCCGCTTG-3'.

\section{Quantitative real-time PCR}

Total RNA was extracted from the NIH3T3, 293ET using the TRIZOL reagent (Invitrogen). TransScript First-Strand cDNA Synthesis SuperMix (TransGen Biotech) was used for reverse transcription. Stem-loop RT-PCR for the mature miRNAs was performed as previously described [27]. The miRNAs and the mRNAs of firefly luciferase, renilla luciferase and GFP were quantified with real-time PCR using SYBR ${ }^{\circledR}$ Premix Ex Taq ${ }^{\mathrm{mm}}$ (TaKaRa). The real-time PCR amplification was performed using $60 \mathrm{~s}$ of denaturation at $95^{\circ} \mathrm{C}$ and 45 cycles of $95^{\circ} \mathrm{C}$ for $5 \mathrm{~s}$ and $60^{\circ} \mathrm{C}$ for $34 \mathrm{~s}$.

\section{Western blotting}

Total cellular protein was separated by $10 \%$ sodium dodecyl sulfate polyacrylamide gel electrophoresis, and the proteins were transferred onto a nitrocellulose membrane. Then, the membrane was probed with rabbit anti-BMAL1 (Cat. sc-8550, Santa Cruz) or mouse antihuman $\beta$-actin antibodies (Cat. A5441, Sigma). After washing, the blots were treated with secondary antibodies conjugated to horseradish peroxidase and developed with ECL plus (Amersham).

\section{Serum shock procedures}

The NIH3T3 cells were synchronized by serum shock as previously described [28]. Briefly, the NIH3T3 cells untreated or transfected with synthetic mir-142-3p mimics or control miRNA mimics were maintained in normal DMEM until the cells reached confluence. At time $=0$, the medium was exchanged with serum-rich medium (supplemented with 50\% FBS) and $2 \mathrm{~h}$ later the medium was replaced with serum-free DMEM. At the indicated times, the cells were harvest and kept in $-70^{\circ} \mathrm{C}$ until the extraction of total RNA.

\section{Computational prediction}

Three miRNA target prediction databases (TargetScan, PicTar and MicroCosm) were used to predict Bmal1/ Clock-targeting miRNAs. The potential targets of mir142-3p and $-5 p$ were predicted using the TargetScan and PicTar algorithms, and the targets predicted by both algorithms were listed in Additional file 4. The conserved E-box (CACGTG) in the upstream regulatory sequence of mir-142 was analyzed using DNAman, and the conservation track was obtained from the UCSC genome browser (http://genome.ucsc.edu/).

\section{Statistical analysis}

Data were expressed as mean \pm SD. Statistical analyses were performed using a two-tailed unpaired t-test to compare data and a P-value less than 0.05 is considered statistically significant.

\section{Additional files}

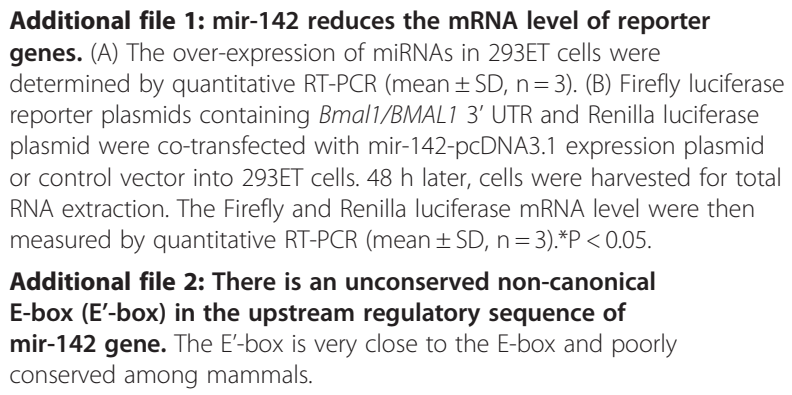

Additional file 3: Screening for miRNAs that can target Clock. (A)

Prediction results for miRNAs that might target clock by TargetScan, PicTar and MicroCosm. miRNAs that were predicted by at least two bioinformatic algorithms were listed in the table. (B) The schematic representation of the luciferase reporter construct containing Clock 3' UTR and the control vector. A $1.9 \mathrm{~kb}$ Clock 3' UTR containing all the binding sites of the candidate miRNAs was cloned downstream the luciferase cassette. (C) Luciferase reporter assay was performed to screen for potential Clock-targeting miRNAs. Data represent mean $\pm S D, n=3$. Two-tailed unpaired t test results are indicated by * for $P<0.05$, relative to cells transfected with control vector.

Additional file 4: Predicting the targets of mir-142-3p and mir-142-5p. The targets predicted by both TargetScan and PicTar were listed in the table. The targets involving in circadian clock are in gray letters.

Additional file 5: Primers for cloning Bmal1/Clock 3' UTR and pre-miRNAs and real-time PCR primers for detecting miRNAs and genes were listed in this table.

\section{Competing interests}

The authors declare that they have no financial or non-financial competing interests.

\section{Authors' contributions}

$X C T$ and $X Z P$ conceived and designed the experiments; $X C T, P Z$ and $L Z$ performed the experiments; XCT, PZ, LZ and XZP analyzed the data; XCT, PZ, $L Z, B Y, H P$ and XZP contributed reagents/materials/analysis tools; XCT and XZP wrote the paper. All authors read and approved the final manuscript.

\section{Acknowledgments}

We thank Dr. Steve Reppert (University of Massachusetts Medical School) and Dr. David Weaver (University of Massachusetts Medical School) for kindly providing the expression plasmids for Clock and Bmal1. Grand support was received from "973" project (No: 2011CBA01104, 2007CB946902 and 2009CB825403), National Sciences Foundation of China (No: 30825023, 31071203) and Program for New Century Excellent Talents (No: NCET-07-0505).

\section{Author details}

${ }^{1}$ State Key Laboratory of Medical Molecular Biology, Institute of Basic Medical Sciences, Chinese Academy of Medical Sciences and Peking Union Medical College, Beijing 100005, China. ${ }^{2}$ Department of Physiology, Institute of Basic Medical Sciences, Chinese Academy of Medical Sciences and Peking Union Medical College, Beijing 100005, China. ${ }^{3}$ Department of Endocrinology, Peking Union Medical Hospital, Chinese Academy of Medical Sciences and Peking Union Medical College, Beijing 100730, China. 
Received: 26 April 2012 Accepted: 23 August 2012

Published: 7 September 2012

\section{References}

1. Storch KF, Paz C, Signorovitch J, Raviola E, Pawlyk B, Li T, Weitz CJ: Intrinsic circadian clock of the mammalian retina: importance for retinal processing of visual information. Cell 2007, 130(4):730-741.

2. Sahar S, Sassone-Corsi P: Metabolism and cancer: the circadian clock connection. Nat Rev Cancer 2009, 9(12):886-896.

3. Lamia KA, Storch KF, Weitz CJ: Physiological significance of a peripheral tissue circadian clock. Proc Natl Acad Sci U S A 2008, 105(39):15172-15177.

4. Bunger MK, Wilsbacher LD, Moran SM, Clendenin C, Radcliffe LA, Hogenesch JB, Simon MC, Takahashi JS, Bradfield CA: Mop3 is an essential component of the master circadian pacemaker in mammals. Cell 2000, 103(7):1009-1017.

5. Gekakis N, Staknis D, Nguyen HB, Davis FC, Wilsbacher LD, King DP, Takahashi JS, Weitz CJ: Role of the CLOCK protein in the mammalian circadian mechanism. Science 1998, 280(5369):1564-1569.

6. Takahashi JS, Hong HK, Ko CH, McDearmon EL: The genetics of mammalian circadian order and disorder: implications for physiology and disease. Nat Rev Genet 2008, 9(10):764-775.

7. Ripperger JA, Schibler U: Rhythmic CLOCK-BMAL1 binding to multiple E-box motifs drives circadian Dbp transcription and chromatin transitions. Nat Genet 2006, 38(3):369-374

8. DeBruyne JP, Weaver DR, Reppert SM: CLOCK and NPAS2 have overlapping roles in the suprachiasmatic circadian clock. Nat Neurosci 2007, 10(5):543-545.

9. Debruyne JP, Noton E, Lambert CM, Maywood ES, Weaver DR, Reppert SM: A clock shock: mouse CLOCK is not required for circadian oscillator function. Neuron 2006, 50(3):465-477.

10. Sangoram AM, Saez L, Antoch MP, Gekakis N, Staknis D, Whiteley A, Fruechte EM, Vitaterna MH, Shimomura K, King DP, et al: Mammalian circadian autoregulatory loop: a timeless ortholog and mPer1 interact and negatively regulate CLOCK-BMAL1-induced transcription. Neuron 1998, 21(5):1101-1113.

11. Preitner N, Damiola F, Lopez-Molina L, Zakany J, Duboule D, Albrecht U, Schibler U: The orphan nuclear receptor REV-ERBalpha controls circadian transcription within the positive limb of the mammalian circadian oscillator. Cell 2002, 110(2):251-260.

12. Xu S, Witmer PD, Lumayag S, Kovacs B, Valle D: MicroRNA (miRNA) transcriptome of mouse retina and identification of a sensory organspecific miRNA cluster. J Biol Chem 2007, 282(34):25053-25066.

13. Cheng HY, Papp JW, Varlamova O, Dziema H, Russell B, Curfman JP, Nakazawa T, Shimizu K, Okamura H, Impey S: microRNA modulation of circadian-clock period and entrainment. Neuron 2007, 54(5):813-829.

14. Nagel R, Clijsters L, Agami R: The miRNA-192/194 cluster regulates the Period gene family and the circadian clock. FEBS J 2009, 276(19):5447-5455.

15. Kadener S, Menet JS, Sugino K, Horwich MD, Weissbein U, Nawathean P, Vagin W, Zamore PD, Nelson SB, Rosbash M: A role for microRNAs in the Drosophila circadian clock. Genes Dev 2009, 23(18):2179-2191.

16. Zhou W, Li Y, Wang X, Wu L, Wang Y: MiR-206-mediated dynamic mechanism of the mammalian circadian clock. BMC Syst Biol 2011, 5(1):141.

17. Shende VR, Goldrick MM, Ramani S, Earnest DJ: Expression and rhythmic modulation of circulating microRNAs targeting the clock gene Bmal1 in mice. PLoS One 2011, 6(7):e22586.

18. Kojima S, Shingle DL, Green CB: Post-transcriptional control of circadian rhythms. J Cell Sci 2011, 124(Pt 3):311-320.

19. Lee $Y$, Chen $R$, Lee HM, Lee C: Stoichiometric relationship among clock proteins determines robustness of circadian rhythms. J Biol Chem 2011, 286(9):7033-7042.

20. Saus E, Soria V, Escaramis G, Vivarelli F, Crespo JM, Kagerbauer B, Menchon JM, Urretavizcaya M, Gratacos M, Estivill X: Genetic variants and abnormal processing of pre-miR-182, a circadian clock modulator, in major depression patients with late insomnia. Hum Mol Genet 2010, 19(20):4017-4025.

21. Huang B, Zhao J, Lei Z, Shen S, Li D, Shen GX, Zhang GM, Feng ZH: miR-142-3p restricts CAMP production in CD4 + CD25- T cells and CD4 + CD25+ TREG cells by targeting AC9 mRNA. EMBO Rep 2009, 10(2):180-185.
22. Sutton GM, Begriche K, Kumar KG, Gimble JM, Perez-Tilve D, Nogueiras $R$, McMillan RP, Hulver MW, Tschop MH, Butler AA: Central nervous system melanocortin-3 receptors are required for synchronizing metabolism during entrainment to restricted feeding during the light cycle. FASEB $J$ 2009, 24(3):862-872.

23. Yan J, Wang H, Liu Y, Shao C: Analysis of gene regulatory networks in the mammalian circadian rhythm. PLoS Comput Biol 2008, 4(10):e1000193.

24. Chao TF, Zhang Y, Yan XQ, Yin B, Gong YH, Yuan JG, Qiang BQ, Peng XZ: [MiR-9 regulates the expression of CBX7 in human glioma]. Zhongguo $Y_{i}$ Xue Ke Xue Yuan Xue Bao 2008, 30(3):268-274.

25. Chen Y, Liu W, Chao T, Zhang Y, Yan X, Gong Y, Qiang B, Yuan J, Sun M, Peng $X$ : MicroRNA-21 down-regulates the expression of tumor suppressor PDCD4 in human glioblastoma cell T98G. Cancer Lett 2008, 272(2):197-205.

26. Gong Y, Yue J, Wu X, Wang X, Wen J, Lu L, Peng X, Qiang B, Yuan J: NSPc1 is a cell growth regulator that acts as a transcriptional repressor of p21Waf1/Cip1 via the RARE element. Nucleic Acids Res 2006, 34(21):6158-6169.

27. Chen C, Ridzon DA, Broomer AJ, Zhou Z, Lee DH, Nguyen JT, Barbisin M, Xu $\mathrm{NL}$, Mahuvakar VR, Andersen MR, et al: Real-time quantification of microRNAs by stem-loop RT-PCR. Nucleic Acids Res 2005, 33(20):e179.

28. Balsalobre A, Damiola F, Schibler U: A serum shock induces circadian gene expression in mammalian tissue culture cells. Cell 1998, 93(6):929-937.

doi:10.1186/1471-2199-13-27

Cite this article as: Tan et al.: Clock-controlled mir-142-3p can target its activator, Bmal1. BMC Molecular Biology 2012 13:27.

\section{Submit your next manuscript to BioMed Central and take full advantage of:}

- Convenient online submission

- Thorough peer review

- No space constraints or color figure charges

- Immediate publication on acceptance

- Inclusion in PubMed, CAS, Scopus and Google Scholar

- Research which is freely available for redistribution 